\title{
Web-based information and support for patients with a newly diagnosed neuroendocrine tumor: a feasibility study
}

\author{
Grietje Bouma $^{1}$ - Lotte D. de Hosson ${ }^{1}$ • Claudia E. van Woerkom ${ }^{1}$ • Hennie van Essen ${ }^{1}$. \\ Geertruida H. de Bock ${ }^{2}$ • Jolien M. Admiraal ${ }^{1}$ • Anna K. L. Reyners ${ }^{1}$ • \\ Annemiek M. E. Walenkamp ${ }^{1}$
}

Received: 7 October 2016 / Accepted: 23 January 2017 / Published online: 9 February 2017

(C) The Author(s) 2017. This article is published with open access at Springerlink.com

\begin{abstract}
Purpose Patients with a neuroendocrine tumor (NET) frequently experience physical and psychosocial complaints. Novel strategies to provide information to optimize supportive care in these patients are of interest. The aim of this study was to examine whether the use of a web-based system consisting of self-screening of problems and care needs, patient education, and self-referral to professional health care is feasible in NET patients and to evaluate their opinion on this.

Methods Newly diagnosed NET patients were randomized between standard care $(n=10)$ or intervention with additional access to the web-based system $(n=10)$ during 12 weeks. Patients completed questionnaires regarding received information, distress, quality of life (QoL), and empowerment. The intervention group completed a semi-structured interview to assess patients' opinion on the web-based system.

Results The participation rate was $77 \%$ (20/26 invited patients) with no dropouts. The use of the web-based system had a negative effect on patients' perception and satisfaction of received information (range Cohen's $d-0.88$ to 0.13 ). Positive effects were found for distress (Cohen's $d 0.75$ ), global QoL (subscale European Organization for Research and Treatment of Cancer (EORTC) QLQ-C30, Cohen's $d$ 0.46), resolving problems with social functioning and finding information (subscales EORTC QLQ-GINET 21, Cohen's $d 0.69$,
\end{abstract}

Annemiek M. E. Walenkamp

a.walenkamp@umcg.nl

1 Department of Medical Oncology, University Medical Center Groningen, University of Groningen, DA11, PO Box 30.001, 9700 RB Groningen, The Netherlands

2 Department of Epidemiology, University Medical Center Groningen, University of Groningen, Groningen, The Netherlands respectively, 1.04), and feeling informed (subscale empowerment questionnaire, Cohen's $d$ 0.51). The interview indicated that the web-based system was of additional value to standard care.

Conclusions Use of this web-based system is feasible. Contradictory effects on informing and supporting NET patients were found and should be subject of further research. Trial registration NCT01849523

Keywords Neuroendocrine tumor · Information · Support . Web-based $\cdot$ Internet

\section{Introduction}

Neuroendocrine tumors (NETs) are rare tumors arising from secretory cells of the neuroendocrine system. Secretion of hormones and/or amines can result in a broad set of symptoms [1,2]. Patients can experience various symptoms as a consequence of the presence of the tumor as well as the release of the hormones secreted by the tumor. Also, side effects of the treatment can bother them. Moreover, diagnosis and treatment of cancer often results in physical, psychological, practical, social, and spiritual concerns [3]. Studies in patients with NETs demonstrate that these concerns lead to lower health-related quality of life (QoL) compared with the general population $[4,5]$. In general, when patients with cancer are properly informed, they experience a better health-related QoL and less depression and anxiety $[6,7]$. In addition, providing information also results in an increased participation of patients in decision-making, greater satisfaction with care, lower levels of distress, and improved sense of control [7-10].

Among patients with cancer, the increasing role of internet as a source of cancer-related information is 
recognized. Web-based patient education and support have become widely accepted [11-13]. We developed a webbased system for NET patients with the aim to examine its feasibility and to evaluate patient's opinion on this. Secondary aims were to explore the effects on patients' perception and satisfaction of received information, distress, health-related QoL, and empowerment. The webbased system allows patients to self-screen for physical and psychosocial problems, to get tailored patient education on reported problems, and if necessary, to refer themselves to care.

\section{Methods}

\section{Participants}

To be eligible for this study, patients had to be newly diagnosed with a NET grade 1 or 2 (according to the World Health Organization 2010 classification) within 3 months before study participation. Any primary site or disease stage was allowed. Patients had to be under surveillance or treatment at the Department of Medical Oncology in the University Medical Center Groningen (UMCG). Additional inclusion criteria were 18 years of age or older and ability to read and write Dutch. Not eligible were patients with an estimated life expectancy of less than 3 months or a second primary tumor with active followup or treatment. All eligible patients were invited to participate. Recruitment occurred at the outpatient clinic during October 2013 to January 2014. The study was approved by the medical ethical committee of the UMCG and registered in ClinicalTrials.gov (NCT01849523). All patients gave written informed consent.

\section{Study procedure}

For detailed description of study procedures, see Appendix 1. Eligible patients were informed about the study by their treating medical oncologist during a follow-up visit or by phone if a follow-up visit was not planned at short notice. Patients were asked if they could be approached for study participation by one of the investigators. If this was allowed, the investigator informed the patient orally after the follow-up visit or by phone. Patients were informed about the aims, study procedures, and feasibility design of this study. Following obtained informed consent, patients were randomized in a 1:1 ratio to the standard care group or the intervention group that received standard care with additional access to the web-based system. At baseline, all patients received questionnaires by mail regarding socio-demographic characteristics, health care/internet use, perception and satisfaction of received information
(EORTC QLQ-INFO25), and QoL (EORTC QLQ-C30, EORTC QLQ-GINET21). At baseline, the standard care group received the paper-and-pencil version of the distress thermometer (DT) and problem list (PL) by mail. The intervention group had to complete the online version of the DT and PL (content identical to the paper-and-pencil version). When questionnaires were completed, the intervention group received instructions how to use the web-based system together with log-in information including a personal username and password. At week 12, all patients received questionnaires by mail regarding frequency of visits to psychosocial and/or allied health care professionals, perception and satisfaction of received information, QoL, and empowerment (Constructs Empowering Outcomes, CEO questionnaire). The standard care group also received the paper-and-pencil version of the DT and PL by mail. The intervention group was reminded to complete the online version of the DT and PL after 12 weeks. At end of the study, the patients assigned to the intervention group were also invited for a semi-structured interview with the investigator regarding their opinion on the webbased system.

Randomization outcome was not blinded and also notified to the treating medical oncologist. In this manner, all patients could discuss their questions about the retrieved information (e.g., from the web-based system, information leaflets) with their treating medical oncologist, oncology nurse, other health care professionals, and/or allied health care workers.

\section{Standard care and study intervention}

Standard care for newly diagnosed patients with a NET during the first visit(s) at the Department of Medical Oncology includes verbal information about the diagnosis, evaluating complaints/problems and a physical examination by their treating medical oncologist. Also, newly diagnosed patients visit an oncology nurse with experience in care for NET patients. All patients receive at diagnosis information leaflets of the Dutch Patient Neuroendocrine Tumor Foundation [14]. During follow-up visits, the medical oncologist evaluates and discusses the general wellbeing, test results, treatment (e.g., options, side effects), answers questions of the patient, and performs a physical examination. If physical and/or psychosocial problems require more in-depth discussion, investigation or treatment, patients can receive a consultation with the oncology nurse or other health care professionals.

In the intervention group, patients received access to the web-based information and support system in addition to standard care during the 12 -week study period. The web-based system incorporates elements of selfscreening for problems and care needs, tailored support in self-help, patient education, and communication with 
a health care professional. Patients screened themselves for psychosocial/physical problems and care needs by filling out the DT and PL. Immediately after completion of the DT, automated personalized feedback was received upon their reported distress score. For example, feedback emphasizes the possibility for a referral to a health care professional for moderate or severe distress. Thereafter, patients received tailored feedback on physical/ psychosocial problems they had reported on the PL. This feedback comprises background information about the problem, advice on how to cope with the problem ("self-help advice") and which health care professional can be consulted when self-help insufficiently alleviates problems. Online completed DT and PL questionnaires with feedback were online saved and coded to ensure that patients could access the feedback and information later.

Also, general information about NETs and diagnostic procedures, treatments, and their side effects is provided by the web-based system. Patients could send an e-mail or request for a telephone call to communicate with the investigator (experienced physician in treating NET patients) in case of questions, problems, or a referral wish. A referral wish to a professional or allied health care worker was arranged by the treating medical oncologist.

\section{Measures}

Patient characteristics including socio-demographic characteristics, use of previous and current psychosocial and/ or allied health care, acquaintance with internet, and use of internet to search cancer-related information were assessed by a self-report questionnaire. Information on disease-related characteristics was collected from medical records.

Participation and dropout rates and reasons for declining study participation and dropout were tracked.

Patients' perceived amount and satisfaction of received information was measured with the Dutch EORTC QLQINFO25 questionnaire, which is a validated questionnaire to evaluate the information received by cancer patients [15]. This questionnaire comprises multi-items grouped into four information provision subscales "perceived receipt of information about the disease," "medical tests," "treatment" and "other care services" and single items on "having received information" in different manners (e.g., written, online, on CD) and on "satisfaction with, amount of and helpfulness of information." In addition, two open questions investigate topics of which patients would like to receive more or less information. All responses are ranged on a 4-point Likert scale, except for four single items that have a "yes/no" scale.

Distress was measured using the validated Dutch DT and PL [3]. The DT consists of a single item asking patients to indicate the amount of overall distress experienced during the past week on an 11-point scale (range "0" no distress-" 10 " extreme distress). The PL identifies existing practical, family/social, emotional, religious/spiritual, and physical problems and was adapted by the investigators according to the issues which are prevalent among and relevant to patients with NETs (in total 31 items). Patients could also add a problem that was not mentioned in the PL. For each reported problem, patients rated the associated experienced amount of distress (range " 0 " no distress-" 10 " extreme distress). The PL ends with the question whether the patient has a referral wish to an oncology nurse, peer NET sufferer group, a psychosocial (psychologist, social or pastoral worker), or allied health care professional (e.g., physical therapist, dietician).

QoL was measured with the validated Dutch EORTC QLQ-C30 (version 3) [16] and Dutch EORTC QLQGINET21 [17]. The EORTC QLQ-C30 measures cancerspecific QoL comprising five functional scales (physical, role, cognitive, emotional, and social), three symptom scales (fatigue, pain and nausea/vomiting), six single symptom items (dyspnea, insomnia, loss of appetite, constipation, diarrhea, and financial impact), and a global health status/QoL scale. Responses are given on a 4point Likert scale (range "1" not at all-“4" very much) with higher scores indicating higher QoL at the functional scales and higher symptom burden for the symptom scales. The global health status/QoL scale is scored on a 7-point scale (range "1" very poor-"7" excellent) with higher scores indicating higher QoL. The EORTC QLQGINET21 is a disease-specific QoL questionnaire for patients with a gastrointestinal NET to supplement the EORTC QLQ-C30 questionnaire and assesses specific disease symptoms, side effects of treatment, body image, disease-related worries, social functioning, communication, and sexuality. Responses are given on a 4-point Likert scale (range "1" not at all-" 4 " very much) with higher scores indicating higher symptom burden for the symptom scales.

Empowerment was measured by the Dutch CEO questionnaire, which is developed and tested in different online support groups including breast cancer patients [11, 18]. In the questionnaire, "online support groups" was modified in "website" for the intervention group and in "standard care" for the control group. The domains "being better informed," "feeling more confident about the relationship with their physician," "improved acceptance of the illness," "feeling more confident about the treatment," and "increased optimism and control over the future" were measured since these domains were expected to be influenced by the web-based system.

Patients' opinion regarding satisfaction with the webbased system was assessed with a semi-structured 
interview by phone with participants in the intervention group. Ten open-ended, both qualitative and quantitative questions were used and when relevant the interviewer probed the participant with follow-up questions to include topics not established beforehand. These questions comprised the following issues: contents, lay out, user-friendliness, perceived usability, usage and future recommendations. The interviews were recorded and afterwards all interviews were transcribed in English. All topics per individual interview related to the purpose of the research were noted and categorized.

\section{Statistical analysis}

The purpose of this study was to explore the feasibility and to evaluate patient's opinion on the web-based system on abovementioned endpoints. Twenty NET patients were recruited and randomized. Descriptive statistics (e.g., means, ranges, frequencies) were calculated for all measures. Missing values for the EORTC questionnaires were handled according to the EORTC guidelines [19]. Raw scores of all scales and items of the EORTC questionnaires were linearly transformed to a score ranging from 0 to 100 [19]. Scores of the different problem/empowerment domains of the PL/CEO questionnaire were calculated by taking the mean of the total scores of the items in each domain. Effect sizes for change scores on each endpoint were determined (Cohen's $d$ ) by using the mean difference between post- and pre-intervention scores of both groups and dividing this difference by the pooled standard deviation. Given the retrospective design of the CEO questionnaire with only a post-intervention measurement, effect sizes of the outcomes of the CEO were calculated by using the mean difference between post-intervention scores of both groups and dividing this difference by the accompanying pooled standard deviation.

All effect sizes are reported so that a positive effect size indicates a desired direction (i.e., improvement of outcome in favor of the intervention group). An effect size (ES) between $0.20-0.50$ is defined as small, between 0.50 and 0.80 as medium and $\geq 0.80$ as large. Analyses were performed using the software package SPSS, version 22 for Windows (SPSS, Inc., Chicago, IL, USA).

\section{Results}

\section{Participants}

Twenty of the 26 invited eligible patients gave informed consent and all patients completed the full study period. Reasons for declining study participation were no access to a computer and internet $(n=2)$, no interest in information and filling out questionnaires $(n=1)$, feeling overburdened $(n=2)$ and not willing to be further confronted with the disease $(n=1)$.

Patient and tumor characteristics are summarized in Table 1. There was a minor imbalance between the intervention and control group regarding age, educational level and primary tumor localization. The intervention group consisted of younger, higher educated patients and more patients with a NET of the lung. During the study, six patients ( $n=1$ in the standard care group) started treatment with a somatostatin analog and two patients (both in standard care group) with everolimus. Three patients ( $n=1$ in the standard care group) underwent curative surgery and two patients in the intervention group underwent palliative surgery during the study period.

\section{Effect of intervention on outcomes}

In the standard care group, one patient was supported by a social worker and another patient by a psychologist during the study period. In the intervention group, two patients received treatment of a physical therapist $(n=2)$ and/or dietician $(n=1)$.

Table 2 presents mean scores of the different subscales of EORTC QLQ-INFO25 at baseline and end of study for the two groups and the accompanying effect sizes. Most outcomes on subscales showed negative effect sizes indicating that the intervention group had less improvement in feeling informed. None of the patients wished to receive less information. At baseline, less patients in the intervention group wished to receive more information (50 vs. $70 \%$ in the intervention respectively standard group).

Baseline and end of study mean scores and accompanying effect sizes of the DT and PL are summarized in Table 3. A medium ES was observed for distress and social problems in favor of the intervention group. Trivial or small effect sizes were observed for problems in the other domains. Few patients experienced religious/ spiritual problems.

Outcomes for QoL measured by EORTC QLQ-C30 and EORTC QLQ-GINET21 questionnaires are depicted in Tables 4 and 5. The intervention group showed a higher increase in QoL (small ES). A moderate ES was observed for cognitive functioning in favor of the standard care group. In contrast, financial difficulties and pain improved more (moderate effect sizes) in the intervention group.

The intervention group showed more improvement on disease-specific problems measured by the EORTC QLQGINET21 questionnaire. For example, large effect sizes were observed for disease-related worries.

Outcomes of the different domains of empowerment are presented in Table 6. The intervention group felt better 
Table 1 Patient and tumor characteristics

\begin{tabular}{|c|c|c|}
\hline & Standard care group $(n=10) N^{\mathrm{a}}$ & Intervention group $(n=10) N^{a}$ \\
\hline Median age in years (range) & $64.0(45-74)$ & $59.5(41-74)$ \\
\hline Gender M/F & $5 / 5$ & $4 / 6$ \\
\hline \multicolumn{3}{|l|}{ Educational level } \\
\hline Lower vocational education & 6 & 3 \\
\hline Intermediate vocational education & 3 & 5 \\
\hline Higher vocational education/university & 1 & 2 \\
\hline \multicolumn{3}{|l|}{ Employment status } \\
\hline Employed & 3 & 4 \\
\hline Unemployed & 7 & 6 \\
\hline \multicolumn{3}{|l|}{ Internet use } \\
\hline In daily life & 8 & 9 \\
\hline For disease purposes & 6 & 7 \\
\hline \multicolumn{3}{|l|}{ Location primary tumor } \\
\hline Lung & 0 & 3 \\
\hline Pancreas & 2 & 2 \\
\hline Jejunum/ileum & 4 & 4 \\
\hline Unknown & 4 & 1 \\
\hline \multicolumn{3}{|l|}{ Disease stage (according to UICC ${ }^{\mathrm{b}}$ ) } \\
\hline Stage I/II & 0 & 2 \\
\hline Stage III & 1 & 2 \\
\hline Stage IV & 9 & 6 \\
\hline Treatment prior to study participation (\%) & $80 \%$ & $50 \%$ \\
\hline Somatostatin analog & 6 & 4 \\
\hline Curative surgery & 0 & 1 \\
\hline Palliative surgery & 4 & 2 \\
\hline Everolimus & 0 & 1 \\
\hline Radiofrequency ablation & 1 & 0 \\
\hline Other $^{\mathrm{c}}$ & 1 & 0 \\
\hline Health care use before diagnosis (\%) & $20 \%$ & $10 \%$ \\
\hline Physical therapist & 1 & 1 \\
\hline Psychologist & 1 & 0 \\
\hline
\end{tabular}

${ }^{\text {a }}$ Data are expressed as number unless noted otherwise

${ }^{\mathrm{b}}$ Union for International Cancer Control

${ }^{\mathrm{c}}$ Proton pump inhibitor

informed (moderate ES) and had more confidence in their treatment (small ES). In contrast, the standard care group scored higher for the domain "acceptance of illness" (small ES) and "optimism and control over the future" (moderate ES).

\section{Qualitative and quantitative semi-structured interview}

To evaluate patient's opinion on the web-based system, all patients in the intervention group were interviewed.

The number of times the web-based system was visited ranged from $2-5$ ( $n=4$ patients), $6-10(n=2), 11-15(n=3)$ to $16-20(n=1)$. Frequently visited parts of the web-based system were "diagnostic procedures" and "treatments." The
DT, specific information about NET, and the part on "diagnostic procedures" and "treatments" were found most useful.

All patients found the web-based system user-friendly.

All but one patient found the information presented by the web-based system understandable. Most patients $(n=6)$ found the provided information by the web-based system "pretty complete," "complete," or "very complete." Suggestions for information to add to the web-based system were "frequently used medications in NET patients other than tumor treatments (e.g., laxatives)," "advice about healthy life style," and "life expectancy."

Seven patients preferred information on their type of tumor only. Two patients were also interested in other types of tumors and enjoyed reading about it. 
Table 2 Perceived information and satisfaction (EORTC QLQINFO25)

\begin{tabular}{|c|c|c|c|c|c|}
\hline \multirow[b]{2}{*}{ Outcome } & \multicolumn{2}{|c|}{ Standard care group $(n=10)$} & \multicolumn{3}{|c|}{ Intervention group $(n=10)$} \\
\hline & Pre M (SD) & Post M (SD) & Pre M (SD) & Post M (SD) & ES \\
\hline \multicolumn{6}{|l|}{ Information about: } \\
\hline Disease & $33.33(21.52)$ & $42.50(18.19)$ & $35.83(21.89)$ & $39.17(19.66)$ & -0.30 \\
\hline Medical tests & $48.89(21.72)$ & $56.67(16.93)$ & $57.78(15.54)$ & $61.11(22.38)$ & -0.22 \\
\hline Treatments & $32.78(13.21)$ & $44.44(15.27)$ & $29.44(17.77)$ & $33.33(16.56)$ & -0.59 \\
\hline Other services & $12.50(14.83)$ & $30.00(30.73)$ & $10.83(15.24)$ & $14.17(16.22)$ & -0.74 \\
\hline Different places of care & $16.67(28.32)$ & $23.33(31.62)$ & $16.67(28.33)$ & $13.33(28.11)$ & -0.17 \\
\hline How to help yourself & $13.33(23.31)$ & $33.33(31.43)$ & $20.00(23.31)$ & $20.00(28.11)$ & -0.88 \\
\hline Received written information & $80.00(42.16)$ & $80.00(42.16)$ & $100.00(0.00)$ & $70.00(48.30)$ & -0.52 \\
\hline Received CD/video & $0.00(0.00)$ & $0.00(0.00)$ & $0.00(0.00)$ & $0.00(0.00)$ & 0.00 \\
\hline Satisfaction with information & $46.67(23.31)$ & $50.00(23.57)$ & $50.00(23.57)$ & $56.67(22.50)$ & 0.13 \\
\hline Wanting to receive more info & $30.00(48.30)$ & $60.00(51.64)$ & $50.00(52.70)$ & $60.00(51.64)$ & -0.28 \\
\hline Wanting to receive less info & $100.00(0.00)$ & $100.00(0.00)$ & $100.00(0.00)$ & $100.00(0.00)$ & 0.00 \\
\hline Helpfullness of information & $46.67(17.21)$ & $56.67(22.50)$ & $46.67(17.21)$ & $56.67(27.44)$ & 0.00 \\
\hline Global score & $38.40(13.30)$ & $48.08(12.82)$ & $43.10(10.51)$ & $43.70(13.66)$ & -1.06 \\
\hline
\end{tabular}

Higher scores mean more/better information and satisfaction

Pre $M$ mean score at baseline, Post $M$ mean score at 12 weeks, $S D$ standard deviation, $E S$ effect size
Most patients $(n=8)$ found the web-based system of additional value. All would recommend this web-based system to peers and believed this should be part of the standard care. Also, several patients considered it of interest for people surrounding the NET patient (e.g., family, friends, general practitioners). All wanted to use the web-based system in the future. Patients would like to use it immediately upon diagnosis.

\section{Discussion}

This is the first study examining whether use of a web-based information and support system is feasible in NET patients. The results suggest that use of this web-based system is feasible. The NET patients appreciated the use of the webbased system and found its use of additional value to standard care. This exploratory study was not powered to detect differences between the two groups.

Use of eHealth-tools is increasing and a goal of the European Commission to improve citizens' health [20]. Given the lack of available eHealth-tools in NET patients, there is an unmet need for a professionally designed webbased information and support system in these patients. Our results provide information to further develop web-based information systems in rare cancer types like NET.

Patients using the web-based information system had less problems with finding adequate information and felt better informed as measured by the EORTC QLQGINET2 1 and CEO. Unexpectedly, the results of the
Table 3 Distress (distress thermometer and problem list)

\begin{tabular}{|c|c|c|c|c|c|}
\hline \multirow[b]{2}{*}{ Outcome } & \multicolumn{2}{|c|}{ Standard care group $(n=10)$} & \multicolumn{3}{|c|}{ Intervention group $(n=10)$} \\
\hline & Pre M (SD) & Post M (SD) & Pre M (SD) & Post M (SD) & ES \\
\hline Distress level & $4.90(2.96)$ & $4.60(3.03)$ & $4.10(3.32)$ & $2.00(1.49)$ & 0.75 \\
\hline \multicolumn{6}{|l|}{ Problems } \\
\hline Practical & $5.10(6.42)$ & $5.60(6.79)$ & $8.20(13.30)$ & $7.80(12.75)$ & 0.16 \\
\hline Social & $0.20(0.63)$ & $0.90(2.03)$ & $2.60(5.23)$ & $2.00(3.27)$ & 0.53 \\
\hline Emotional & $17.00(22.40)$ & $12.50(14.65)$ & $20.10(19.20)$ & $12.20(14.40)$ & 0.20 \\
\hline Spiritual & $0.00(0.00)$ & $0.00(0.00)$ & $0.50(1.58)$ & $0.40(0.97)$ & 0.19 \\
\hline Physical & $18.10(11.83)$ & $11.80(10.62)$ & $16.90(11.40)$ & $11.70(11.41)$ & -0.10 \\
\hline Global score & $40.40(34.63)$ & $30.80(27.12)$ & $48.30(43.33)$ & $34.10(39.86)$ & 0.18 \\
\hline
\end{tabular}

Higher scores mean more distress/problems

Pre $M$ mean score at baseline, Post $M$ mean score at 12 weeks, $S D$ standard deviation, $E S$ effect size 
Table 4 Quality of life (EORTC QLQ-C30)

\begin{tabular}{lllllll}
\hline & \multicolumn{2}{l}{ Standard care group $(n=10)$} & & Intervention group $(n=10)$ & \\
\cline { 2 - 3 } Outcome & Pre M (SD) & Post M (SD) & & Pre M (SD) & Post M (SD) & ES \\
\hline Global quality of life & $59.17(20.95)$ & $58.33(24.53)$ & & $65.83(26.77)$ & $70.83(17.67)$ & 0.46 \\
Functioning & & & & & \\
Physical & $73.33(23.09)$ & $76.67(24.19)$ & & $79.33(27.30)$ & $80.67(20.23)$ & -0.15 \\
Role & $61.67(28.38)$ & $61.67(32.44)$ & & $66.67(36.00)$ & $70.00(21.94)$ & 0.15 \\
Emotional & $67.50(36.74)$ & $73.33(25.99)$ & & $70.00(32.20)$ & $78.33(20.11)$ & 0.11 \\
Cognitive & $78.33(27.27)$ & $83.33(24.85)$ & & $78.33(20.86)$ & $70.00(23.31)$ & -0.61 \\
Social & $68.33(14.59)$ & $75.00(26.35)$ & & $73.33(31.62)$ & $78.33(20.86)$ & -0.08 \\
Symptoms & & & & & & \\
Dyspnea & $23.33(27.44)$ & $20.00(23.31)$ & & $36.67(33.15)$ & $30.00(24.60)$ & 0.15 \\
Insomnia & $16.67(23.57)$ & $20.00(28.11)$ & & $23.33(27.44)$ & $20.00(28.11)$ & 0.21 \\
Appetite loss & $23.33(35.31)$ & $10.00(22.50)$ & & $20.00(35.83)$ & $13.33(23.31)$ & -0.30 \\
Constipation & $6.67(14.05)$ & $10.00(22.50)$ & & $16.67(32.39)$ & $13.33(23.31)$ & 0.14 \\
Diarrhea & $23.33(22.50)$ & $23.33(38.65)$ & & $16.67(32.39)$ & $16.67(32.39)$ & 0.00 \\
Financial difficulties & $10.00(16.10)$ & $23.33(27.44)$ & $26.67(43.89)$ & $23.33(35.31)$ & 0.63 \\
Fatigue & $45.56(31.62)$ & $37.78(34.03)$ & $33.33(36.29)$ & $33.33(28.21)$ & -0.40 \\
Nausea and vomiting & $13.33(20.49)$ & $1.67(5.27)$ & & $18.33(33.75)$ & $10.00(21.08)$ & -0.12 \\
Pain & $26.67(33.52)$ & $25.00(26.35)$ & & $35.00(38.05)$ & $21.67(20.86)$ & 0.52 \\
\hline
\end{tabular}

Higher scores for quality of life and functioning represent higher quality of life and level of functioning. Higher scores for symptoms represent a higher level of symptomatology

Pre $M$ mean score at baseline, Post $M$ mean score at 12 weeks, $S D$ standard deviation, $E S$ effect size
EORTC QLQ-INFO25 demonstrated that use of the webbased system resulted in less improvement of feeling informed and satisfaction of received information. The EORTC QLQ-INFO25 is not specifically developed for patients with an NET, which could potentially explain the observed difference between questionnaires. However, it is possible that a web-based system results in feeling worse informed and more depressed. In contrast to face-to-face contact with health care providers, visiting a web-based system does not offer the possibility to discuss feelings of insecurity in person or to ask questions after having read disturbing information about their disease. Also, the intervention group had less acceptance of illness and less control and optimism regarding the future at the end of the

Table 5 Quality of life (EORTC QLQ-GINET21)

\begin{tabular}{|c|c|c|c|c|c|c|c|c|c|}
\hline \multirow[b]{2}{*}{ Outcome } & \multicolumn{4}{|c|}{ Standard care group $(n=10)$} & \multicolumn{5}{|c|}{ Intervention group $(n=10)$} \\
\hline & Pre M (SD) & $N$ & Post M (SD) & $N$ & Pre M (SD) & $N$ & Post M (SD) & $N$ & $\mathrm{ES}$ \\
\hline Endocrine symptoms & $27.78(19.07)$ & 10 & $22.22(14.81)$ & 10 & $25.56(21.63)$ & 10 & $13.33(11.48)$ & 10 & 0.35 \\
\hline Gastrointestinal symptoms & $28.00(22.40)$ & 10 & $27.33(14.55)$ & 10 & $30.67(29.18)$ & 10 & $24.00(21.36)$ & 10 & 0.34 \\
\hline Treatment-related symptoms & $14.44(18.22)$ & 5 & $15.54(18.56)$ & 5 & $14.44(18.22)$ & 5 & $23.33(16.81)$ & 5 & -0.40 \\
\hline Problems social functioning & $28.89(15.89)$ & 10 & $35.56(12.62)$ & 10 & $42.22(28.11)$ & 10 & $38.89(24.15)$ & 10 & 0.69 \\
\hline Disease-related worries & $33.95(26.42)$ & 9 & $42.59(31.18)$ & 9 & $45.00(28.50)$ & 10 & $32.22(22.03)$ & 10 & 0.92 \\
\hline Pain muscles/bone & $16.67(23.57)$ & 10 & $16.67(23.57)$ & 10 & $37.50(45.21)$ & 8 & $37.50(33.03)$ & 8 & 0.00 \\
\hline Problems sexual functioning & $9.52(16.27)$ & 7 & $14.29(26.23)$ & 7 & 38.89 (38.97) & 6 & $33.33(42.16)$ & 6 & 0.52 \\
\hline Problems receiving information & $13.33(17.21)$ & 10 & $20.00(23.31)$ & 10 & $23.33(27.44)$ & 10 & $10.00(16.10)$ & 10 & 1.04 \\
\hline Problems body image & $20.00(17.21)$ & 10 & $13.33(17.21)$ & 10 & $3.33(10.54)$ & 10 & $6.67(14.05)$ & 10 & -0.60 \\
\hline
\end{tabular}

Higher scores mean more or worse symptoms/problems

Pre $M$ mean score at baseline, $N$ number of patients, Post $M$ mean score at 12 weeks, $S D$ standard deviation, ES effect size 
Table 6 Empowerment (CEO)

\begin{tabular}{llll}
\hline Outcome & $\begin{array}{l}\text { Standard care group }(n=10) \\
\text { Mean (SD) }\end{array}$ & $\begin{array}{l}\text { Intervention group }(n=10) \\
\text { Mean (SD) }\end{array}$ & ES \\
\hline Feeling informed & $13.60(3.86)$ & $15.30(3.02)$ & 0.51 \\
Confidence in relationship physician & $37.06(6.78)$ & $36.20(10.17)$ & -0.10 \\
Confidence in treatment & $16.60(4.35)$ & $18.60(4.30)$ & 0.46 \\
Acceptance of illness & $17.60(2.12)$ & $16.40(5.54)$ & -0.29 \\
Optimism and control over future & $25.00(4.69)$ & $22.50(3.87)$ & -0.58 \\
\hline
\end{tabular}

Higher scores represent better empowerment for each outcome

Mean mean score (at 12 weeks), $S D$ standard deviation, $E S$ effect size

study period. A randomized controlled study examining different types of internet support groups in breast cancer patients, also reported negative effects on depression and anxiety in the internet support patients who received additional information and support of other breast cancer patients [21]. Being provided with more information can possibly be confronting for patients with cancer which may result in an increase of negative thoughts and worse future perspectives.

This study had a small sample size due to the feasibility design. Younger age and higher educational level are associated with more internet use for health care information [22]. Therefore, the intervention group may have had a more critical view on the received information. As a result of these small sample sizes despite randomization, the intervention group was younger, higher educated and comprised three lung NET patients. The DT/PL is an element of the web-based system and therefore the intervention group had to fill out the DT/PL online instead of a paper-and-pencil version. Since the content of the online DT/PL is identical to the paper-and-pencil version, we do not expect that this has affected the outcome.

The results of the current study have given insight into the existing worries and problems of NET patients, but also into the needs and expectations of information and support. This has enabled us to develop a sufficiently powered randomized controlled trial investigating an adapted web-based system for patients with both new and a longer known diagnosis of NET (ClinicalTrials. gov Identifier NCT02472678). Mainly based on the results of the semi-structured interview, adaptations have been made to the web-based system, such as adding new information (e.g., information about commonly used medication by NET patients and average life expectancy), "truths and hoaxes" about NET, and experiences of patients with different types of NET with their disease. Hopefully, firm conclusions about effects of this webbased system can be drawn after finishing this study. If the web-based system is proven effective after finishing this study, it will become available for all NET patients in the Netherlands.
Acknowledgments This study was supported by an educational grant of Ipsen to the institute. The sponsor had no involvement in any part of the study.

Compliance with ethical standards The study was approved by the medical ethical committee of the UMCG and registered in ClinicalTrials. gov (NCT01849523). All patients gave written informed consent.

Conflict of interest This study was supported by an educational grant of Ipsen to the institute. The sponsor had no involvement in any part of the study. All primary data are in control of the authors and are allowed to be reviewed by the journal if necessary.

\section{Appendix 1. Extensive description study procedures}

Eligible patients were informed by their treating medical oncologist about the study during a follow-up visit or by phone if the next follow-up visit was planned beyond 2 months. Patients who were interested in study participation were subsequently approached by one of the investigators and received study information. Following informed consent, patients were randomized in a 1:1 ratio to standard care group or the intervention group that received standard care with additional access to the web-based system. Randomization was performed by e-mail from the investigator to the central data manager of the Department of Medical Oncology (UMCG) who is not involved in the recruitment or treatment of the patients. For allocation to the standard care or intervention group, a computer-generated randomization list was used. The allocation sequence was concealed from the investigator. The investigator was informed by e-mail to which group the patient was assigned. The investigator informed the patient by phone about the randomization outcome and further process. Also, the treating medical oncologist was notified about the randomization outcome. After randomization, all patients received questionnaires by mail about socio-demographic characteristics, health care/internet use, perception and satisfaction of received information (EORTC QLQ-INFO25), and quality of life (EORTC QLQ-C30, EORTC QLQ-GINET21) as baseline measurement. The standard care group also received the paper-and-pencil version of the distress thermometer (DT) and problem list (PL). After the investigator had collected these completed questionnaires, the intervention group received via 
their preferred route an e-mail or letter with a short introduction of the web-based system together with log-in information including a personal username and password. These patients were asked to complete the online DT and PL within 1 week after having received the log-in information (baseline measure DT and PL). At week 12, all patients received questionnaires by mail about health care use, perception and satisfaction of received information, quality of life, and empowerment (Constructs Empowering Outcomes questionnaire) as end of study measurement. The standard care group also received the paper-and-pencil version of the DT and PL. The intervention group was reminded by an e-mail/letter to complete the online version of the DT and PL after 12 weeks. Patients assigned to the intervention group were also requested to complete a semistructured interview regarding their opinion on the web-based system by phone.

Open Access This article is distributed under the terms of the Creative Commons Attribution-NonCommercial 4.0 International License (http:// creativecommons.org/licenses/by-nc/4.0/), which permits any noncommercial use, distribution, and reproduction in any medium, provided you give appropriate credit to the original author(s) and the source, provide a link to the Creative Commons license, and indicate if changes were made.

\section{References}

1. Oberg KE (2012) The management of neuroendocrine tumours: current and future medical therapy options. Clin Oncol 24:282-293

2. Ramage JK, Ahmed A, Ardill J, Bax N, Breen DJ, Caplin ME et al (2012) Guidelines for the management of gastroenteropancreatic neuroendocrine (including carcinoid) tumours (NETs). Gut 61:6-32

3. Tuinman MA, Gazendam-Donofrio SM, Hoekstra-Weebers JE (2008) Screening and referral for psychosocial distress in oncologic practice: use of the Distress Thermometer. Cancer 113:870-878

4. Frojd C, Larsson G, Lampic C, von Essen L (2007) Health related quality of life and psychosocial function among patients with carcinoid tumours. a longitudinal, prospective, and comparative study. Health Qual Life Outcomes 5:18

5. Haugland T, Vatn MH, Veenstra M, Wahl AK, Natvig GK (2009) Health related quality of life in patients with neuroendocrine tumors compared with the general Norwegian population. Qual Life Res 18:719-726

6. Husson O, Mols F, van de Poll-Franse LV (2011) The relation between information provision and health-related quality of life, anxiety and depression among cancer survivors: a systematic review. Ann Oncol 22:761-772
7. Davies NJ, Kinman G, Thomas RJ, Bailey T (2008) Information satisfaction in breast and prostate cancer patients: implications for quality of life. Psychooncology 17:1048-1052

8. Cox A, Jenkins V, Catt S, Langridge C, Fallowfield L (2006) Information needs and experiences: an audit of UK cancer patients. Eur J Oncol Nurs 10:263-272

9. Mallinger JB, Griggs JJ, Shields CG (2005) Patient-centered care and breast cancer survivors' satisfaction with information. Patient Educ Couns 57:342-349

10. Rehnberg G, Absetz P, Aro AR (2001) Women's satisfaction with information at breast biopsy in breast cancer screening. Patient Educ Couns 42:1-8

11. van Uden-Kraan CF, Drossaert CH, Taal E, Shaw BR, Seydel ER, van de Laar MA (2008) Empowering processes and outcomes of participation in online support groups for patients with breast cancer, arthritis, or fibromyalgia. Qual Health Res 18:405-417

12. Samoocha D, Bruinvels DJ, Elbers NA, Anema JR, van der Beek AJ (2010) Effectiveness of webbased interventions on patient empowerment: a systematic review and meta-analysis. J Med Internet Res 12:e23

13. Bouma G, Admiraal JM, de Vries EG, Schroder CP, Walenkamp AM, Reyners AK (2015) Internetbased support programs to alleviate psychosocial and physical symptoms in cancer patients: a literature analysis. Crit Rev Oncol Hematol 95:26-37

14. Stichting NET-groep informatie over neuro-endocriene kanker. Available at: www.net-kanker.nl

15. Arraras JI, Greimel E, Sezer O, Chie WC, Bergenmar M, Costantini A et al (2010) An international validation study of the EORTC QLQ-INFO25 questionnaire: an instrument to assess the information given to cancer patients. Eur J Cancer 46:2726-2738

16. Aaronson NK, Ahmedzai S, Bergman B, Bullinger M, Cull A, Duez NJ et al (1993) The European Organization for Research and Treatment of Cancer QLQ-C30: a quality-of-life instrument for use in international clinical trials in oncology. J Natl Cancer Inst 85:365-376

17. Yadegarfar G, Friend L, Jones L, Plum LM, Ardill J, Taal B et al (2013) Validation of the EORTC QLQGINET21 questionnaire for assessing quality of life of patients with gastrointestinal neuroendocrine tumours. Br J Cancer 108:301-310

18. van Uden-Kraan CF, Drossaert CH, Taal E, Seydel ER, van de Laar MA (2009) Participation in online patient support groups endorses patients' empowerment. Patient Educ Couns 74:61-69

19. Manuals EORTC. Available at: http://groups.eortc.be/qol/manuals. Accessed October 1, 2015

20. European Commission. Available at: http://ec.europa.eu/health/ ehealth. Accessed January 21, 2016

21. Lepore SJ, Buzaglo JS, Lieberman MA, Golant M, Greener JR, Davey A (2014) Comparing standard versus prosocial internet support groups for patients with breast cancer: a randomized controlled trial of the helper therapy principle. J Clin Oncol 32:4081-4086

22. van de Poll-Franse LV, van Eenbergen MC (2008) Internet use by cancer survivors: current use and future wishes. Support Care Cancer 16:1189-1195 